\title{
Efeito da Temperatura da Água nas Respostas Cardiovasculares Durante a Caminhada Aquática
}

Effect of Water Temperature in Cardiovascular Responses During Aquatic Walking

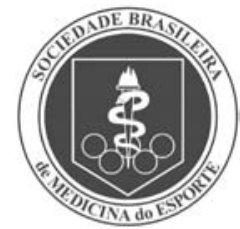

Artigo Original

Angélica Cristiane Ovando ${ }^{1,2}$

Heloisa Meinke Eickhoff

Jonathan Ache Dias²

Eliane Roseli Winkelmann

1. Universidade do Noroeste do Estado do Rio Grande do Sul -

Unijuí - Departamento de Ciências da Saúde. Curso de Fisioterapia. ljuí, Rio Grande do Sul (RS), Brasil. 2. Universidade do Estado de Santa Catarina - Udesc - Centro de Ciências da Saúde e do Esporte Cefid - Programa de Pós-Graduação em Ciências do Movimento Humano. Florianópolis, Santa Catarina (SC), Brasil.

\section{Endereço para correspondência:} Angélica Cristiane Ovando Rua Desembargador Pedro Silva, 2.202, bloco 7, apto. 33, Bairro Coqueiros - 88080-700 Florianópolis, SC - Brasil. E-mail: angecris@yahoo.com.br

Submetido em: 05/08/2008 Versão final recebida em: 30/06/2009 Aceito em: 05/10/2009

\begin{abstract}
RESUMO
O objetivo deste estudo foi verificar o efeito da temperatura da água nas respostas cardiovasculares: frequência cardíaca (FC), pressão arterial sistólica (PAS) e pressão arterial diastólica (PAD), durante a caminhada aquática em três temperaturas $\left(29^{\circ} \mathrm{C}, 33^{\circ} \mathrm{C}\right.$ e $\left.37^{\circ} \mathrm{C}\right)$. Participaram do estudo 10 homens, com média de idade de $23,2 \pm$ 2,25 anos, massa corporal de $78,4 \pm 4,01 \mathrm{~kg}$ e estatura de $1,774 \pm 0,017 \mathrm{~m}$. Os participantes realizaram caminhadas aquáticas durante $30 \mathrm{~min}$, a uma cadência controlada de 55 passos/minuto, na altura do processo xifoide, em dias diferentes para cada temperatura, sendo as respostas cardiovasculares monitoradas nos minutos 5, 10, 20 e 30. Apesar do efeito principal de 75,4\% ( $p \leq 0,001)$ da temperatura da água sobre a variação da FC, também foi identificado um efeito do tempo de exercício de $91,8 \%(p=0,001)$. A FC aumentou gradativamente no decorrer da caminhada, especialmente na temperatura de $37^{\circ} \mathrm{C}(71,3 \pm 8,4$ para $114,6 \pm 4,4)$; ao final da caminhada foi maior que os $29^{\circ} \mathrm{C}(p \leq 0,01)$ e $33^{\circ} \mathrm{C}(p \leq 0,05)$; estas últimas não apresentaram diferença entre si. A temperatura parece ter tido pouco efeito sobre a PAS, visto que apenas nos minutos 20 e 30 foi maior aos $33^{\circ} \mathrm{C}$ comparada com a de $29^{\circ} \mathrm{C}(\mathrm{p} \leq 0,05)$. A PAD sofreu efeito da temperatura, diminuindo gradativamente no decorrer da caminhada, especialmente na temperatura de $37^{\circ} \mathrm{C}(70,0 \pm 5,0$ para $40,0 \pm 2,5)$; apresentou diferença significativa em relação às temperaturas de $29^{\circ} \mathrm{Ce} 33^{\circ} \mathrm{C}(\mathrm{p} \leq 0,05)$, as quais não mostraram diferença entre si. Considerando o efeito da temperatura da água sobre a FC e a PAD durante a caminhada aquática, sugere-se que, quando piscinas terapêuticas forem utilizadas para realização de caminhada na água, a escolha da temperatura da água seja considerada, recomendando-se valores entre $29^{\circ} \mathrm{C}$ e $33^{\circ} \mathrm{C}$ para menor estresse cardiovascular.
\end{abstract}

Palavras-chave: imersão, exercício, frequência cardíaca, pressão arterial.

\begin{abstract}
The purpose of this study was to investigate the effect of water temperature in cardiovascular responses: heart rate (HR), systolic blood pressure (SBP) and diastolic blood pressure (DBP), while walking in water at different temperatures $\left(29^{\circ} \mathrm{C}, 33^{\circ} \mathrm{C}\right.$ e $37^{\circ} \mathrm{C}$ ). Ten men, mean age $23.2 \pm 2.25$ years, mean weight $78.4 \pm 4.01 \mathrm{~kg}$, mean stature $1.774 \pm 0.017 \mathrm{~m}$, participated in the study. Each individual underwent water walking in different days for each temperature during $30 \mathrm{~min}$, with a controlled cadence of 55 steps per minute, at a depth in the xiphoid process. During aquatic walking, the HR and blood pressure (BP) were measured at the $5^{\text {th }}, 10^{\text {th }}, 20^{\text {th }}$, and $30^{\text {th }}$ minute of walking. Despite the $75.4 \%$ ( $\left.p \leq 0.001\right)$ main effect of water temperature on the HR, we also identified a significant effect of $91.8 \%$ ( $p=0.001$ ) of exercise time. HR increased most during exercise in water at $37^{\circ} \mathrm{C}(71.3 \pm 8.4$ to $114.6 \pm 4.4)$, showing significant difference compared to $29^{\circ} \mathrm{C}(p \leq 0.01)$ and $33^{\circ} \mathrm{C}(p \leq 0.05)$ temperatures, which were not different from each other. Water temperature seemed to have little effect on the $S B P$, we have only identified differences between $29^{\circ} \mathrm{C}$ and $33^{\circ} \mathrm{C}$ temperatures in the $20^{\prime}$ e $30^{\prime}$ minutes $(p \leq 0.05)$. DBP has decreased at all temperatures studied, and it was more expressive in $37^{\circ} \mathrm{C}(70.0 \pm 5.0$ to $40.0 \pm 2.5)$ showing significant difference compared to $29^{\circ} \mathrm{C}$ and $33^{\circ} \mathrm{C}(p \leq 0.05)$ temperatures, which were not different from each other. Considering the effect of water temperature on HR and DBP during water walking, it is suggested that water temperature should be considered when walking in water, and temperatures between 29 and $33 \mathrm{C}$ are recommended for less cardiovascular strain during water walking.
\end{abstract}

Keywords: immersion, exercise, heart rate, blood pressure.

\section{INTRODUÇÃO}

A prática de atividades físicas aquáticas torna-se cada vez mais popular, incluindo tanto indivíduos saudáveis quanto portadores de diversas patologias. Dentre as modalidades de exercícios aquáticos, a caminhada tem sido utilizada em atividades de reabilitação, terapêuticas e também em programas de condicionamento específicos, sendo particularmente útil para indivíduos com lesões nos membros inferiores. Tal modalidade de exercício destaca-se ainda pelo fato de não envolver habilidades especiais, além de poder ser realizada nas mais variadas condições de saúde do indivíduo ${ }^{(1,2)}$.
Na reabilitação em ambiente aquático é necessário conhecer, além das sutilezas do meio, as alterações fisiológicas produzidas nas diversas modalidades de atividades, durante e após a imersão em diferentes condições de temperatura da água. O acompanhamento da forma pela qual a frequência cardíaca (FC) e a pressão arterial (PA) reagem em diferentes temperaturas da água durante o exercício pode ser útil na apreciação do estresse cardiovascular provocado pela temperatura da água. Tais variáveis permitem o entendimento das respostas cardiovasculares agudas ao exercício, que consistem em uma série complexa de ajustes para for- 
necer aos músculos em atividade um suprimento adequado de sangue, ao mesmo tempo em que se dissipa calor e se mantém um aporte de nutrientes necessários a órgãos vitais como o cérebro e o coração(3).

Estudos têm relatado o efeito do meio aquático sobre o comportamento da FC; alguns deles relatam a ocorrência de taquicardia ${ }^{(4-9)}$, outros afirmam que não há alterações ${ }^{(10)}$ e a maior parte dos estudos revela ocorrência de bradicardia ${ }^{(9,11-20)}$. No que diz respeito ao comportamento da PA, foram encontrados poucos estudos e as respostas são contraditórias. De acordo com a literatura revisada, a pressão arterial sistólica (PAS) pode aumentar, diminuir ou mesmo permanecer inalterada ${ }^{(8,9,21)}$, enquanto a pressão arterial diastólica (PAD) tem mostrado redução durante a imersão em várias temperaturas ${ }^{(8,9,21)}$. A caminhada aquática também tem sido objeto de alguns estudos enfocando as respostas cardiorrespiratórias e metabólicas ${ }^{(7,21,22)}$, mas poucos apontam as diferenças dessas variáveis em diferentes temperaturas. Além disso, nos estudos encontrados, a caminhada é realizada em esteira rolante submersa, o que não é comum na prática clínica diária.

A FC é muito utilizada para prescrição e monitorização da intensidade do exercício, em função de suas relações com o consumo de oxigênio e com a intensidade de trabalho( ${ }^{(23)}$, sendo um método de fácil mensuração. No entanto, sua aplicação nos programas de exercícios aquáticos pode ser equivocada, dada a influência do meio aquático sobre o sistema cardiovascular, especialmente quando se desconsidera a temperatura da água na qual a atividade será realizada. Conforme Sheldahl et al.(24), a FC para o treinamento na água não deve ser a mesma dos exercícios em terra, visto que as alterações na FC em ambiente aquático são causadas pela temperatura da água e/ou pela hipervolemia central. A monitorização e o controle das respostas cardiovasculares durante o exercício aquático em diferentes temperaturas tornam-se fundamentais na condução segura das atividades propostas, especialmente quando se trabalha com indivíduos cujas condições clínicas permitem pensar em risco cardiovascular aumentado.

Considerando a presente preocupação no que se refere a um possível estresse provocado por atividade aeróbica em piscinas terapêuticas aquecidas, este estudo teve como objetivo verificar o efeito da temperatura da água nas respostas cardiovasculares (FC e PA) de homens jovens e saudáveis durante a caminhada aquática sem a utilização de esteira, em três diferentes temperaturas $\left(29^{\circ} \mathrm{C}, 33^{\circ} \mathrm{C}\right.$ e $\left.37^{\circ} \mathrm{C}\right)$, que se assemelham àquelas comumente utilizadas na hidroterapia. Dessa forma, buscou-se identificar o ambiente com menor estresse cardiovascular para a realização de caminhada aquática em intensidade apropriada para a reabilitação, especialmente no caso de lesões dos membros inferiores.

\section{MÉTODOS}

\section{Sujeitos}

Participaram do presente estudo 10 sujeitos do sexo masculino, não atletas, com 23,2 $\pm 2,2$ anos, massa corporal de 78,4 $\pm 4,0 \mathrm{~kg}$, estatura de $1,77 \pm 0,02 \mathrm{~m} \mathrm{e} \mathrm{VO}_{2 \max }$ de $51,709 \pm 4,714 \mathrm{ml} \cdot \mathrm{kg}^{-1} \cdot \mathrm{min}^{-1}$, avaliado por teste ergoespirométrico, mostrando-se um grupo homogêneo em relação à aptidão física. O grupo foi selecionado a partir dos seguintes critérios de inclusão: deveriam ser homens, adaptados ao meio líquido, com faixa etária entre 20 e 25 anos e estatura entre 1,75 e 1,80m. Como critério de exclusão foi estabelecido que não devessem apresentar lesões musculoesqueléticas no ato da coleta e nem diagnóstico de doenças cardiovasculares. Os sujeitos foram convidados a participar do estudo, informados sobre os procedimentos de forma verbal e escrita, e em seguida assinaram o termo de consentimento livre e esclarecido. Os procedimentos foram aprovados previamente pelo comitê de ética em pesquisa local.

\section{Procedimentos da coleta de dados}

0 presente estudo foi realizado em uma piscina terapêutica $(6 \times 4 \times 1,5 \mathrm{~m})$. Uma semana antes da coleta foi realizado um pré-experimento, onde os indivíduos foram submetidos a todo o protocolo. Esse momento serviu para definir a cadência apropriada (passos/minuto), com velocidade confortável semelhante à utilizada em situações de reabilitação e que pudesse ser mantida em um mesmo ritmo durante 30 minutos de forma cômoda. Estabelecida a cadência (55 passos/min), os sujeitos treinaram a marcha em cadência controlada durante 30 minutos, reproduzindo todo o procedimento de coleta de dados que teria inicio na semana seguinte. Após o pré-experimento, os sujeitos participaram de três sessões, cada uma em temperatura diferente a $33^{\circ} \mathrm{C}, 29^{\circ} \mathrm{C}$, e $37^{\circ} \mathrm{C}$, nessa ordem, em diferentes dias, com intervalo de uma semana entre cada avaliação. Todos os sujeitos foram avaliados seguindo essa mesma sequência de temperaturas. A cadência foi controlada utilizando como metrônomo um sinal sonoro gravado em um compact disk (CD).

As avaliações ocorreram sempre às $15 \mathrm{~h}$. Foi solicitado que os participantes não realizassem exercícios por 48 horas e também se abstivessem do consumo de bebidas que pudessem conter cafeína e/ou álcool depois das $21 \mathrm{~h}$ do dia anterior ao do teste. No local da avaliação, os indivíduos permaneceram em repouso na posição sentada durante 10 minutos. Em seguida foi verificada, de cada sujeito, a PA com um esfigmomanômetro (Heidji, Brasil) e um estetoscópio (Diasyst, Brasil), e a FC com um oxímetro de pulso (Nonin Onyx 9500, EUA), na posição sentada, em repouso. Todos os instrumentos de medida estavam devidamente calibrados. A aferição da pressão arterial foi realizada por um avaliador experiente, cuja reprodutibilidade na situação de repouso e de exercício foi determinada previamente, estabelecendo-se um erro técnico de medida intra-avaliador menor que $2 \%$. A temperatura ambiente durante a coleta dos dados permaneceu entre 22 e $28^{\circ} \mathrm{C}$ de acordo com termômetro digital da marca Incoterm (resolução de $0,1^{\circ} \mathrm{C}$ ). Para medida da temperatura da água foi utilizado um termômetro químico de líquido da marca Incoterm, com resolução de $0,5^{\circ} \mathrm{C}$.

Os valores encontrados no minuto de pré-imersão foram considerados os de FC e PA em repouso. Após essa verificação, cada sujeito foi instruído a entrar lentamente na água, que estava à altura do processo xifoide até o ponto que foi demarcado como sendo o inicio da caminhada, onde permaneceu em repouso por 30 segundos, ouvindo o sinal sonoro do CD com o ritmo da caminhada. Após 30 segundos, foi dado o comando verbal para que iniciasse a marcha. Os parâmetros de FC e PA foram monitorados nos minutos 5, 10, 20 e 30. Para a mensuração desses, cada sujeito permaneceu com a articulação do ombro esquerdo em abdução de $\pm 90^{\circ}$ com o cotovelo estendido na borda da piscina, mantendo marcha estacionária na frequência do metrônomo enquanto eram verificadas a FC e PA. Adicionalmente, ao final de cada minuto estabelecido nas três diferentes temperaturas, foi apresentada a escala de Borg ${ }^{(25)}$ de 6-20 pontos e cada sujeito indicou o nível de esforço percebido. Ao final dos 30 minutos, cada sujeito deixou a piscina e permaneceu fora da água, em repouso na posição sentada, e a FC e PA foram novamente monitoradas no quinto e $10^{\circ}$ minuto após a saída da piscina.

\section{ANÁLISE ESTATÍSTICA}

A estatística descritiva constituiu-se da apresentação da tendência central dos dados, média ou mediana, e da dispersão com a utilização do desvio padrão (DP) ou intervalo interquartil (IIQ). Os dados da FC apresentaram distribuição normal e os da PA não, de acordo com o teste de Shapiro-Wilk. Como estatística inferencial para análise da variável FC foi utilizado o teste ANOVA fatorial $3 \times 4$ com duas variáveis 
independentes dentre participantes, a temperatura com três níveis $\left(29^{\circ} \mathrm{C}, 33^{\circ} \mathrm{C}\right.$ e $\left.37^{\circ} \mathrm{C}\right)$ e os minutos em imersão (tempo de caminhada) a quatro níveis $\left(5^{\prime}, 10^{\prime}, 20^{\prime}\right.$ e $\left.30^{\prime}\right)$. O teste $t$ pareado foi utilizado como post-hoc para verificar os efeitos simples tanto da temperatura quanto do tempo de caminhada. Os efeitos principais da temperatura e do tempo de caminhada sobre a PA foram analisados com o teste não paramétrico de Friedman. Posteriormente, para verificar os efeitos simples, foi utilizado o teste de Wilcoxon. Os testes foram realizados com o software SPSS versão 14.0, adotando-se um nível de significância de $5 \%(p \leq 0,05)$.

\section{RESULTADOS}

Verificada a condição de esfericidade e homogeneidade das variâncias dos valores da FC, o teste ANOVA fatorial identificou um efeito principal de $91,8 \%$ ( $F=89,217$; $d f=1 ; p \leq 0,001)$ do tempo de caminhada (minutos) e 75,4\% ( $F=24,527 ; d f=1 ; p=0,001)$ da temperatura sobre a variação da variável FC. Não foi encontrada interação entre o tempo de exercício e temperatura sobre a FC.

Com relação ao efeito principal da temperatura sobre a PA, a tabela 1 apresenta a comparação da PAS e da PAD entre as temperaturas em cada minuto da caminhada. No entanto, como foi utilizado um teste não paramétrico, não foi possível definir o tamanho deste efeito.

Tabela 1. Comparação da pressão arterial entre as três condições de temperatura em cada minuto da caminhada aquática.

\begin{tabular}{|c|c|c|c|c|}
\hline minuto & PA & $\aleph^{2}$ & df & $p$ \\
\hline \multirow{2}{*}{ pré-imersão } & PAS & 4,88 & 2 & $p=0,087$ \\
\hline & PAD & 0,46 & 2 & $p=0,792$ \\
\hline \multirow{2}{*}{$5^{\prime}$} & PAS & 4,22 & 2 & $p=0,121$ \\
\hline & PAD & 3,69 & 2 & $p=0,157$ \\
\hline \multirow{2}{*}{$10^{\prime}$} & PAS & 5,35 & 2 & $p=0,069$ \\
\hline & PAD & 9,50 & 2 & $p=0,009^{*}$ \\
\hline \multirow{2}{*}{$20^{\prime}$} & PAS & 6,54 & 2 & $p=0,038^{*}$ \\
\hline & PAD & 8,22 & 2 & $p=0,016^{*}$ \\
\hline \multirow{2}{*}{$30^{\prime}$} & PAS & 6,00 & 2 & $p=0,050^{*}$ \\
\hline & PAD & 13,27 & 2 & $p=0,001^{*}$ \\
\hline
\end{tabular}

$\aleph^{2}=$ estatística de Friedman. $\mathrm{df}=$ graus de liberdade. $\mathrm{PAS}=$ pressão arterial sistólica. $\mathrm{PAD}=$ pressão arterial diastólica. ${ }^{*} p \leq 0,05$

O comportamento da FC, PAS e PAD nas três condições de temperatura e em diferentes minutos da caminhada é mostrado na figura 1, onde a FC está apresentada com média e DP e a PAS e PAD, com mediana e IIQ. A figura 1 apresenta também os efeitos simples (comparação aos pares) da temperatura sobre a FC e PA.

A tabela 2 apresenta a diferença dos valores da FC e PA entre os minutos de pré-imersão e o final da caminhada (30').

A figura 2 apresenta a mediana dos valores da percepção de esforço subjetiva de todos os sujeitos em cada minuto, avaliada pela escala de Borg $^{(25)}$ nas três condições de temperatura.

\section{DISCUSSÃO}

No presente estudo o exercício de caminhada realizado pode ser considerado de baixa intensidade para esse grupo de jovens do sexo masculino, confirmado pela média da FC apresentada que não se aproximou da FC submáxima ou máxima, e pela percepção subjetiva do esforço (figura 2). O comportamento da FC durante o exercício foi crescente nas três temperaturas, no entanto, em $37^{\circ} \mathrm{C}$ observou-
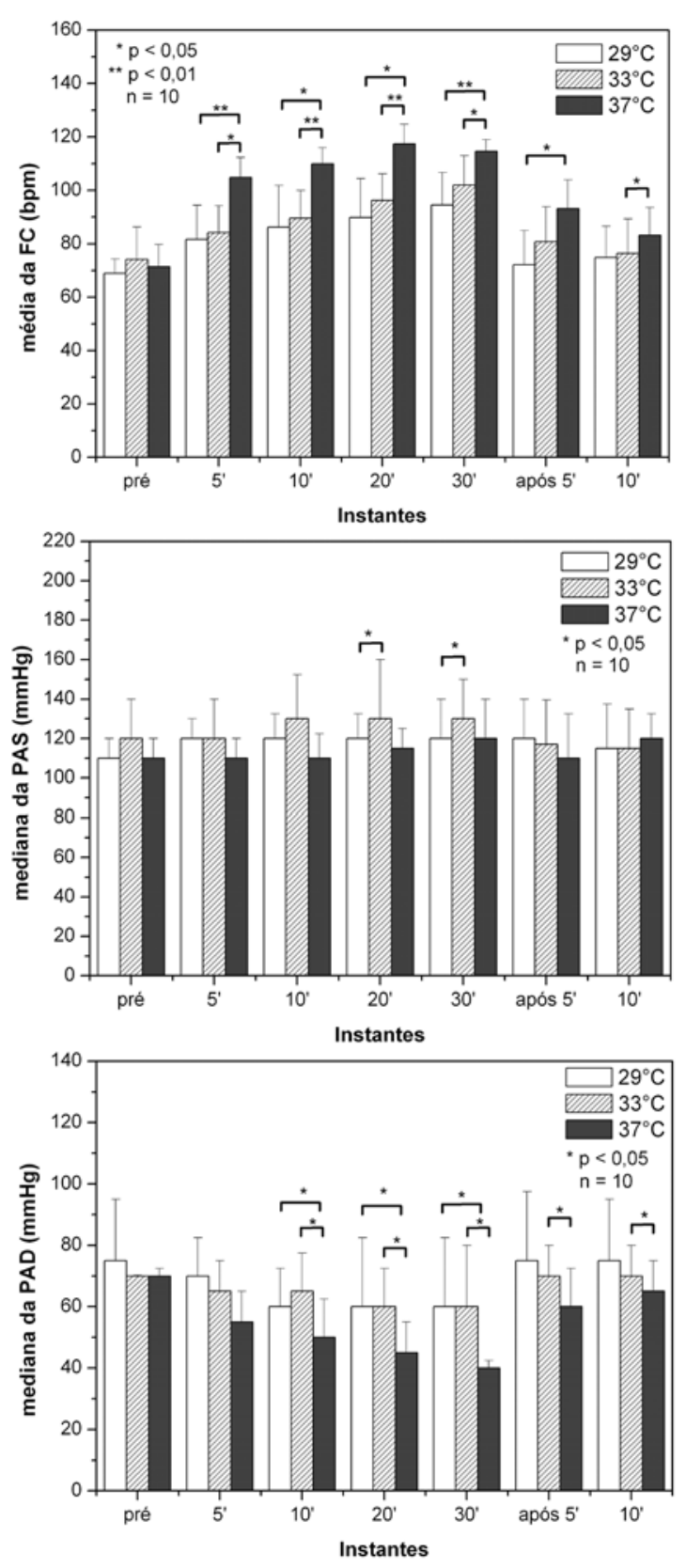

Figura 1. Comportamento da FC, PAS e PAD durante caminhada aquática em três condições de temperatura.

Tabela 2. Comparação da FC e PA entre a pré-imersão e 30' nas três condições de temperatura.

\begin{tabular}{|c|c|c|c|c|c|}
\hline Variável & Temperatura & Pré & $30^{\prime}$ & $\mathrm{t}$ & sig \\
\hline \multirow{3}{*}{$\begin{array}{c}\text { FC } \\
\text { (bpm) }\end{array}$} & $29^{\circ} \mathrm{C}$ & $69,5 \pm 5,4$ & $94,6 \pm 12,1$ & $-5,470$ & $0,001 *$ \\
\hline & $33^{\circ} \mathrm{C}$ & $74,1 \pm 12,2$ & $102,3 \pm 13,2$ & $-6,809$ & $0,001 *$ \\
\hline & $37^{\circ} \mathrm{C}$ & $71,3 \pm 8,4$ & $114,6 \pm 4,4$ & $-15,934$ & $0,001^{*}$ \\
\hline \multirow{4}{*}{$\begin{array}{c}\text { PAS } \\
(\mathrm{mmHg})\end{array}$} & & $\begin{array}{c}\text { Mediana } \\
\pm \text { IIQ }\end{array}$ & $\begin{array}{c}\text { Mediana } \\
\pm \text { IIQ }\end{array}$ & z & sig \\
\hline & $29^{\circ} \mathrm{C}$ & $110,0 \pm 10,0$ & $120,0 \pm 20,0$ & $-1,706$ & 0,088 \\
\hline & $33^{\circ} \mathrm{C}$ & $120,0 \pm 20,0$ & $130,0 \pm 20,0$ & $-2,309$ & $0,021^{*}$ \\
\hline & $37^{\circ} \mathrm{C}$ & $110,0 \pm 10,0$ & $120,0 \pm 10,0$ & $-1,508$ & 0,132 \\
\hline \multirow{3}{*}{$\begin{array}{c}\text { PAD } \\
(\mathrm{mmHg})\end{array}$} & $29^{\circ} \mathrm{C}$ & $75,0 \pm 20,0$ & $60,0 \pm 22,5$ & $-2,739$ & $0,006^{*}$ \\
\hline & $33^{\circ} \mathrm{C}$ & $70,0 \pm 2,5$ & $60,0 \pm 20,0$ & $-2,220$ & $0,026^{*}$ \\
\hline & $37^{\circ} \mathrm{C}$ & $70,0 \pm 5,0$ & $40,0 \pm 2,5$ & $-2,871$ & $0,004^{*}$ \\
\hline
\end{tabular}

$\mathrm{t}=$ estatística do teste $t$ pareado. $\mathrm{z}=$ estatística do teste de Wilcoxon. $\mathrm{FC}=$ frequência cardíaca, $\mathrm{PAS}=$ pressão arterial sistólica. PAD = pressão arterial diastólica. $\| \mathrm{Q}=$ intervalo interquartil. ${ }^{*} p \leq 0,05$ 


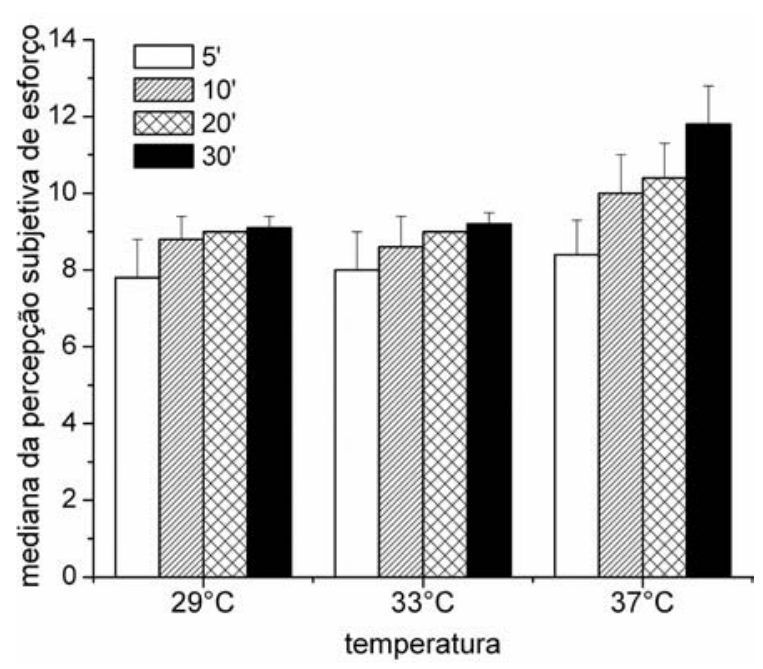

Figura 2. Percepção subjetiva de esforço avaliada pela escala de Borg durante a caminhada aquática nas três condições de temperatura.

se maior elevação da FC durante a caminhada; os valores médios ao final de $30^{\prime}$ foram $21 \%$ maiores que em $29^{\circ} \mathrm{C}$ e $12 \%$ maiores que em $33^{\circ} \mathrm{C}$. Além disso, os indivíduos relataram sensação de desconforto e maior dificuldade para realizar a caminhada na temperatura de $37^{\circ} \mathrm{C}$, o que pode ser conferido pelo esforço percebido (figura 2). O estudo de Weston et al. ${ }^{(26)}$ apresenta importantes reflexões no que diz respeito ao efeito da imersão em repouso na temperatura de $37^{\circ} \mathrm{C}$. Os autores observaram taquicardia e aumento da temperatura corporal central de $0,5^{\circ} \mathrm{C}$. Dessa forma, a observação de $\mathrm{FC}$ mais elevada após exercício de caminhada nessa mesma temperatura no presente estudo parece consistente com o efeito no repouso. Os autores ${ }^{(26)}$ sugerem que a principal contribuição para essa taquicardia tenha sido maior taxa de despolarização do nodo sinoatrial que ocorre em temperaturas corporais elevadas. Tal observação poderia contribuir para explicar a FC mais elevada na temperatura de $37^{\circ} \mathrm{C}$ no presente estudo. Outra hipótese para o aumento da $\mathrm{FC} \mathrm{em} 37^{\circ} \mathrm{C}$ no presente estudo poderia estar relacionada com gasto energético maior nessa temperatura, uma vez que a FC reflete o gasto energético e a intensidade metabólica durante o exercício e essa resposta é, em parte, dependente da temperatura da água ${ }^{(1)}$.

O comportamento semelhante dos valores médios da FC de repouso nas três diferentes etapas da coleta de dados indica que a dimensão das alterações encontradas na FC durante a caminhada foi provocada pelas diferentes temperaturas de água utilizadas. Alguns estudos abordaram o efeito da temperatura da água no comportamento da FC durante o exercício. McArdle et al.(.27) observaram diminuição da FC em temperaturas mais baixas da água comparadas com mais elevadas durante exercício em cicloergômetro aquático. Shimizu et al. ${ }^{(28)}$, ao comparar caminhada de cinco minutos em esteira subaquática em temperaturas de 25,30 e $35^{\circ} \mathrm{C}$, observaram que a $\mathrm{FC}$ teve maior aumento durante o exercício a $35^{\circ} \mathrm{C}$ e, de acordo com os autores, esse comportamento pode ser explicado pela necessidade aumentada de fluxo sanguíneo para a pele na temperatura mais elevada. No mesmo estudo, não houve diferença entre o $\mathrm{VO}_{2 \max }$ durante a caminhada nas três diferentes temperaturas.

Os valores de FC encontrados no presente estudo corroboram os estudos supracitados, onde o mesmo exercício realizado em um ambiente aquático com temperatura mais elevada apresenta maiores valores da FC. Considerando que o exercício em meio aquático normalmente é prescrito com base na FC e esforço subjetivo, a temperatura da água deve ser considerada ao utilizar esse indicador de intensidade de esforço no meio aquático. Ainda, deve ser considerado o fato de, em temperaturas termoneutras $\left(32^{\circ} \mathrm{C}\right.$ e $\left.33^{\circ} \mathrm{C}\right)$, a FC ser menor no exercício aquático comparado com o terrestre ${ }^{(1)} \mathrm{e}$, em temperaturas mais elevadas, a FC ser maior ou igual à do exercício terrestre ${ }^{(21,28)}$. Conforme observado no estudo de Hall et al. ${ }^{(21)}$ e Shimizu et al.(28), em temperaturas mais elevadas, para uma mesma intensidade de exercício, a FC foi maior; no entanto, o consumo de $\mathrm{O}_{2}$ permaneceu o mesmo. Embora o presente estudo não tenha avaliado $\circ \mathrm{VO}_{2}$ durante a realização da caminhada, a observação de estudos prévios permite afirmar que a prescrição de exercício para condicionamento cardiorrespiratório em piscina terapêutica aquecida acima de $36^{\circ} \mathrm{C}$, baseada na $\mathrm{FC}$, poderia ser equivocada, uma vez que o indivíduo poderia atingir sua $\mathrm{FC}$ alvo de treinamento com menor intensidade de exercício. De acordo com Graef e Kruel(29), a escala de Borg parece ser uma opção adequada para o controle da intensidade de exercícios aquáticos, considerando-se as recomendações inerentes a sua aplicação.

Quanto ao comportamento da PAS, foi possível identificar um efeito da temperatura na variação da mesma; no entanto, as diferenças encontradas foram somente entre as temperaturas de $29^{\circ} \mathrm{C}$ e $33^{\circ} \mathrm{C}$ nos $20^{\prime}$ e $30^{\prime}$ de caminhada (figura 1). Quando comparado o valor pré-imersão com o de $30^{\prime}$ (tabela 2), somente foi observada diferença significativa na temperatura de $33^{\circ} \mathrm{C}$, indicando que o tempo de exercício não teve efeito sobre a PAS nas demais temperaturas. Tais achados permitem afirmar que a temperatura teve pouco efeito sobre essa variável, pois em relação à temperatura $37^{\circ} \mathrm{C}$ não foram observadas diferenças significativas.

De acordo com Sramek et al. ${ }^{(30)}$, as mudanças na PA induzidas pela imersão não são uniformes. Em seu estudo, a PAS durante a imersão em repouso de uma hora diminuiu em média $11 \%$ nas temperaturas de $20^{\circ} \mathrm{C}$ e $32^{\circ} \mathrm{C}$. Em outro estudo( ${ }^{(9)}$, com imersão em repouso durante 30 minutos em três condições de temperaturas diferentes $\left(29^{\circ} \mathrm{C}, 33^{\circ} \mathrm{C}\right.$ e $37^{\circ} \mathrm{C}$ ), a PAS também diminuiu em todas elas. Aumento da PAS durante exercício em meio aquático é identificado no trabalho de Hall et al. ${ }^{(21)}$, no qual se observou elevação após cinco minutos de caminhada em esteira submersa, nas temperaturas de $28^{\circ} \mathrm{C}$ e $36^{\circ} \mathrm{C}$, sendo que o aumento maior foi observado na de $36^{\circ} \mathrm{C}$. Em outro estudo(31), foi avaliada a regulação cardiovascular durante exercício em bicicleta ergométrica na terra e em ambiente aquático na temperatura $32^{\circ} \mathrm{C}$. Foram encontrados valores semelhantes da PAS, comparando-se água e terra, nas mais variadas intensidades de exercício.

Em relação à PAD, foi detectado no presente estudo que durante a caminhada ocorreu diminuição gradativa da mesma em todas as temperaturas (figura 1), principalmente na $37^{\circ} \mathrm{C}$. O comportamento decrescente da PAD durante a caminhada é muito semelhante ao do durante o repouso, observado no estudo de Ovando et al.(9), onde foram utilizadas as mesmas temperaturas da água do presente estudo, e a PAD teve comportamento decrescente em todas as temperaturas estudadas. Esse fato pode indicar que a diminuição da PAD durante o exercício, neste estudo, pode ter ocorrido em parte em função da imersão em si, que provoca diversas alterações no sistema circulatório(1). A temperatura, entretanto, também teve efeito sobre a PAD no presente trabalho, uma vez que esta mostrou comportamento diferente entre as três temperaturas estudadas, com valores mais baixos especialmente na de $37^{\circ} \mathrm{C}$, totalizando redução de $42,9 \%$ quando comparada com valores pré-imersão (tabela 2). Comportamento semelhante é observado no trabalho de Hall et al. ${ }^{(21)}$, que ao comparar as respostas cardiorrespiratórias entre duas temperaturas $\left(28^{\circ} \mathrm{C}\right.$ e $\left.36^{\circ} \mathrm{C}\right)$, somente em $36^{\circ} \mathrm{C}$ encontraram diminuição significativa da PAD. Segundo os mesmos autores, a vasodilatação resultante da imersão em água aquecida aumenta o débito cardíaco e o fluxo sanguíneo para a pele e, dessa forma, aumenta a PAS e reduz a PAD. Além disso, foi demonstrado que a resistência 
vascular sistêmica diminui durante a imersão em água aquecida, tanto em pessoas jovens como em pacientes com insuficiência cardíaca ${ }^{(32)}$. A medida da resistência vascular sistêmica não foi realizada neste estudo, mas uma redução da mesma causada pelo aumento da temperatura da água explicaria a maior diminuição da PAD na temperatura de $37^{\circ} \mathrm{C}$.

Embora seja coerente que resultados de indivíduos jovens e saudáveis não sejam extrapolados para populações especiais de pacientes, um exemplo de comportamento da PA e FC é apresentado no presente estudo, e algumas questões devem ser consideradas quanto ao uso de piscina terapêutica aquecida para a prática de caminhada aquática. A caminhada na água, com intensidade semelhante à utilizada durante situações de reabilitação, com cadência de 55 passos por minuto, mostrou-se uma atividade fácil nas temperaturas de $29^{\circ} \mathrm{C}$ e $33^{\circ} \mathrm{C}$ (figura 2), ficando entre relativamente fácil e ligeiramente cansativa na temperatura de $37^{\circ} \mathrm{C}$ para essa população. A FC apresentou valores mais elevados em temperatura de $37^{\circ} \mathrm{C}$. Além disso, a PAD reduziu-se consideravelmente durante caminhada em temperatura mais elevada, gerando mal-estar nos sujeitos. Quanto à PAS, esta parece não ser influenciada pela temperatura mais aquecida, mas estudos em populações especiais poderiam trazer respostas mais seguras.

Algumas limitações, no entanto, devem ser consideradas, pois podem ter comprometido, em parte, a interpretação dos resultados. No presente trabalho não foi realizada a aferição da temperatura corporal central, ou mesmo da temperatura da pele, o que dificulta algumas reflexões que melhor explicariam o comportamento das variáveis do estudo frente às variações de temperatura aquática. Os autores também chamam a atenção para o fato do não balanceamento da amostra quanto à ordem de temperatura da água, visto que todos os indivíduos foram submetidos à mesma ordem de temperatura aquática para a realização da caminhada. Na tentativa de evitar alteração das variáveis FC e PA em função do aprendizado da tarefa, houve o cuidado de realizar um estudo pré-experimento, com repetição de todo o protocolo de coleta, familiarizando os sujeitos ao ambiente aquático e ao ritmo de caminhada imposta. Além disso, em um estudo semelhante onde os in-

\section{REFERÊNCIAS BIBLIOGRÁFICAS}

1. Cureton KJ. Respostas fisiológicas ao exercício na água. In: Routi RG, Morris DM, Cole A J, editores. Reabilitação aquática. Săo Paulo: Manole, 2000;43-63.

2. Hall J, Swinkels A, Briddon J, McCabe CS. Does aquatic exercise relieve pain in adults with neurologic or musculoskeletal disease? A systematic review and meta-analysis of randomized controlled trials. Arch Phys Med Rehabil. 2008;89:873-83.

3. Polito MD, Farinatti PTV. Respostas de frequência cardíaca, pressão arterial e duplo-produto ao exercício contra-resistência: uma revisão da literatura. Rev Port Cien Desp. 2003;3:79-91.

4. Denison DM, Wagner PD, Kingaby GL, West JB. Cardiorespiratory responses to exercise in air and underwater J Appl Physiol. 1972;33:426-30.

5. Johnson BL, Stromme SB, Adamezyk JW, Tennoe KO. Comparison of oxygen uptake and heart rate during exercises on land and in water. Phys Ther. 1977;57:273-8.

6. Kenny GP, Giesbrecht GG, Thoden JS. A comparison of human thermoregulatory response following dynamic exercise and warm-water immersion. Eur J Appl Physiol. 1996;74:336-41.

7. Whitley JD, Schoene LL. Comparison of heart rate responses: water walking versus treadmill walking. Phys Ther. 1987;67:1501-4

8. Allison TG, Maresh CM, Armstrong LE. Cardiovascular responses in a whirlpool bath at $40^{\circ} \mathrm{C}$ versus usercontrolled water temperatures. Mayo Clin Proc. 1998;73:210-5.

9. Ovando AC, Winkelmann ER, Eickhoff HM. O comportamento da frequência cardíaca e da pressão arteria durante imersăo aquática a diferentes temperaturas em repouso. Fisioter Bras. 2006;7:260-7.

10. Begin R, Epstein M, Sackner MA, Levinson R, Dougherty R, Duncan D. Effects of water immersion to the neck on pulmonary circulation and tissue volume in man. J Appl Physiol. 1976;40:293-9.

11. Frangolias DD, Rhodes EC. Maximal and ventilatory threshold responses to treadmill and water immersion running. Med Sci Sports Exerc. 1995;27:1007-13.

12. Denadai BS, Rosas R, Denadai MLDR. Limiar aeróbio e anaeróbio na corrida aquática: comparaçăo com os valores obtidos na corrida em pista. Rev Bras Ativ Fis Saúde. 1997;2:23-8.

13. Town GP, Bradley SS. Maximal metabolic responses of deep and shallow water running in trained runners. Med Sci Sports Exerc. 1991;23:238-41.

14. Kruel LFM, Moraes EZC, Ávila AOV, Sampedro RMF. Alterações fisiológicas e biomecânicas em indivíduos praticando exercícios de hidroginástica dentro e fora d'água. Rev Kinesis. 2001; nº especial:104-29.

15. Kruel LFM, Tartaruga LAP, Dias AC, Silva RC, Picanço PSP, Rangel AB. Frequência cardíaca durante imersão no meio aquático. Rev Fit Perfor. 2002;1:46-51

16. Risch WD, Koubenec HJ, Beckmann U, Lange S, Gauer OH. The effect of graded immersion on heart volume, cen- divíduos foram submetidos a atividades aquáticas em três temperaturas distintas, em dias distintos, foi observado que a ordem de avaliação em temperatura diferente, ou na terra, não alterou os resultados ${ }^{(28)}$.

\section{CONCLUSÃO}

O presente estudo possibilitou verificar o efeito da temperatura da água nas respostas cardiovasculares de homens jovens e saudáveis durante a caminhada aquática; na de $37^{\circ} \mathrm{C}$ os indivíduos apresentaram valores aumentados da FC, além de maiores alterações na PAD nessa mesma temperatura, demonstrando que temperaturas mais altas parecem ter maior efeito sobre o sistema cardiovascular. Nossos resultados não foram consistentes para demonstrar o efeito da temperatura sobre a PAS. Embora a temperatura aquecida da água seja desejável para aliviar a dor e promover relaxamento muscular durante a reabilitação aquática, sugere-se que, quando a piscina terapêutica for utilizada para exercício de caminhada contínua e prolongada em homens jovens, a escolha da temperatura da água seja considerada, recomendando-se valores entre $29^{\circ} \mathrm{C}$ e $33^{\circ} \mathrm{C}$, evitando assim queda excessiva da PAD e sensação de cansaço. Ainda, ao estabelecer a intensidade da caminhada por meio da FC, deve ser considerada, entre outros fatores, a temperatura da água.

Apesar de a temperatura não ter demonstrado efeito sobre a PAS nessa população, sugere-se a realização de estudos que avaliem o efeito da temperatura da água durante a caminhada aquática sobre as respostas cardiovasculares de populações especiais como idosos, indivíduos hipertensos e/ou com outras patologias cardíacas, visto que o meio aquático tem sido uma escolha frequente para exercícios físicos e reabilitação em tais populações.

\section{AGRADECIMENTOS}

Agradecemos à Clínica de Fisioterapia Fisio \& Vida de ljuí/RS por ceder a piscina para a realização das avaliações.

Todos os autores declararam não haver qualquer potencial conflito de interesses referente a este artigo. tral venous pressure, pulmonary blood distribution, and heart rate in man. Pflügers Archiv. 1978:374:115-8. 17. Graef F, Tartaruga L, Alberton C, Kruel L. Freqüência cardíaca em homens imersos em diferentes temperaturas de água. Rev Port Cien Desp. 2006;5:266-73.

18. Cider A, Svealv BG, Tang MS, Schaufelberger M, Andersson B. Immersion in warm water induces improvement in cardiac function in patients with chronic heart failure. Eur J Heart Fail. 2006;8:308-13.

19. Schmid JP, Noveanu M, Morger C, Gaillet R, Capoferri M, Anderegg M, et al. Influence of water immersion, water gymnastics and swimming on cardiac output in patients with heart failure. Heart. 2007;93:722-7.

20. Svedenhag J, Seger J. Running on land and in water: comparative exercise physiology. Med Sci Sports Exerc. 1992;24:1155-60

21. Hall J, Macdonald IA, Maddison PJ, O'Hare JP. Cardiorespiratory responses to underwater treadmill walking in healthy females. Eur J Physiol Occup Physiol. 1998;77:278-84.

22. Fujishima K, Shimizu T. Body temperature, oxygen uptake and heart rate during walking in water and on land at an exercise intensity based on RPE in elderly men. Appl Human Sci. 2003;22:83-8.

23. American College of Sports Medicine. ACSM's Guidelines for Exercise Testing and Prescription. 7th ed. Baltimore: Lippincott Williams \& Wilkins, 2006.

24. Sheldahl LM, Buskirk ER, Loomis $J$ L. Effect of head out water immersion on cardiorespiratory response to dynamic exercise. J Am Coll Cardiol. 1987;10:1254-8.

25. Borg GAV. Psychophysical bases of perceived exertion. Med Sci Sports Exerc. 1982;14:377-81.

26. Weston CFM, O'Hare JP, Evans JM, Corrall RJM. Haemodynamic changes in man during immersion in water at different temperatures. Clin Sci. 1987;73:613-6.

27. McArdle WD, Magel JR, Lesmes GR, Pechar GS. Metabolic and cardiovascular adjustment to work in air and water at 18,25 and 33०C.J Appl Physiol. 1976;40:85-90.

28. Shimizu T, Kosaka M, Fujishima K. Human thermoregulatory responses during prolonged walking in water at 25,30 , and $35^{\circ} \mathrm{C}$. Eur J Appl Physiol 1998:78:473-8.

29. Graef FI, Kruel LFM. Freqüência cardíaca e percepção subjetiva do esforço no meio aquático: diferenças em relação ao meio terrestre e aplicações na prescriçăo do exercício - uma revisăo. Rev Bras Med Esporte. 2006;12:221-8.

30. Sramek P, Simeckova M, Jansky L, Savlikova J, Vybiral S. Human physiological responses to immersion into water of different temperatures. Eur J Appl Physiol. 2000;81:436-42.

31. Christie JL, Sheldahl LM, Tristani FE, Wann LS, Sagar KB, Levandoski SG, et al. Cardiovascular regulation during head-out water immersion exercise. J Appl Physiol. 1990;69:657-64.

32. Meyer K, Bucking J. Exercise in heart failure: should aqua therapy and swimming be allowed? Med Sci Sports Exerc. 2004;36:2017-23. 